\title{
Hepatitis C virus infection contributes to impregnation of markers of immune inhibition: potential preludes underlying viral latency and persistence
}

\author{
Muttiah Barathan', Mohamed Rosmawati ${ }^{2}$, Jamuna Vadivelu', Marie Larsson ${ }^{3}$, Vijayakumar Velu ${ }^{4}$, Saeidi Alireza', \\ Li Yen Chang ${ }^{1}$, Esaki Muthu Shankar ${ }^{{ }^{*}}$
}

From 2nd International Science Symposium on HIV and Infectious Diseases (HIV SCIENCE 2014)

Chennai, India. 30 January - 1 February 2014

\section{Background}

Hepatitis $\mathrm{C}$ virus (HCV) represents one of the persistent viral infections afflicting humankind, and a significant proportion of chronic $\mathrm{HCV}$ disease progresses over time through liver fibrosis, cirrhosis and hepatocellular carcinoma (HCC). One potential mechanism underlying the chronic disease is believed to be viral escape from immune surveillance via upregulation of inhibitory molecules on immune cells by $\mathrm{HCV}$. We investigated the diverse expression of various inhibitory molecules in $\mathrm{PBMCs}$ of healthy non-HCV controls and chronically $\mathrm{HCV}$ infected patients.

\section{Methods}

The expression of inhibitory molecules on PBMCs was investigated in chronic $\mathrm{HCV}$ infected patients relative to healthy non-HCV controls using standard immunological and molecular methods. The serum levels of indoleamine 2, 3 deoxygenase (IDO) and cyclooxygenase- 2 (COX-2) were also investigated.

\section{Results}

The gene expression profile of chronically HCV infected patients was significantly different from control individuals. Our results showed upregulation of TIM-3 $(p \leq 0.01), \mathrm{PD}-1(p \leq 0.01)$, FOXP-3 $(p \leq 0.01)$, BLIMP-1 $(p \leq 0.01), \mathrm{CD} 160(p \leq 0.01), \mathrm{CTLA}-4(p \leq 0.01)$, TRAIL

\footnotetext{
* Correspondence: shankarem@um.edu.my

${ }^{1}$ Tropical Infectious Disease Research and Education Center (TIDREC), Department of Medical Microbiology, Faculty of Medicine, University of Malaya, Kuala Lumpur, Malaysia

Full list of author information is available at the end of the article
}

$(p \leq 0.01)$, BTLA $(p \leq 0.01)$ and LAG-3 $(p \leq 0.01)$ with fold change of $1.3,0.4,14.6,0.87,6.6,0.4,14.7,10.9$ and 2.5 respectively in chronically $\mathrm{HCV}$ infected patients. The plasma IDO and COX-2 levels were significantly higher $(p=0.001)$ in chronically HCV infected subjects relative to healthy control.

\section{Conclusion}

The upregulation of inhibitory molecules on PBMCs in chronically HCV infected patients suggest the contribution of these molecules to immune cells impairment in $\mathrm{HCV}$ infection. Viral persistence and eventual progression following potential evasion of the host immune armory via viral impregnation of inhibitory immune biosignatures in $\mathrm{HCV}$ disease pathogenesis warrants further elucidation.

\section{Authors' details \\ ${ }^{1}$ Tropical Infectious Disease Research and Education Center (TIDREC), Department of Medical Microbiology, Faculty of Medicine, University of Malaya, Kuala Lumpur, Malaysia. ${ }^{2}$ Department of Medicine, Faculty of Medicine, University of Malaya, Kuala Lumpur, Malaysia. ${ }^{3}$ Department of Clinical and Experimental Medicine, Division of Molecular Virology, Laboratory 1, Level 13, Linköping University, 58185 Linköping, Sweden. ${ }^{4}$ Department of Microbiology and Immunology, Emory Vaccine Center, 954 Gatewood Road, Atlanta, Georgia, USA.}

Published: 27 May 2014

doi:10.1186/1471-2334-14-S3-P3

Cite this article as: Barathan et al:: Hepatitis C virus infection contributes to impregnation of markers of immune inhibition: potential preludes underlying viral latency and persistence. BMC Infectious Diseases 2014 14(Suppl 3):P3. 